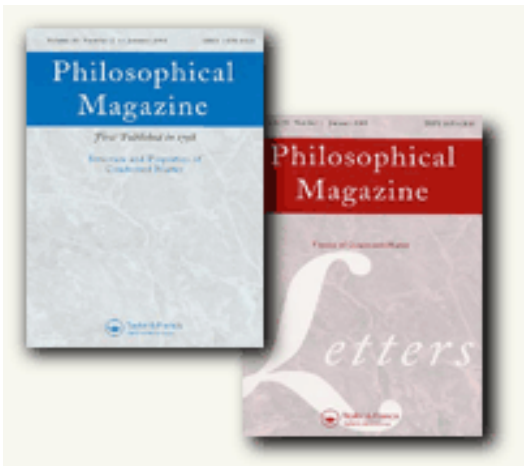

\title{
Decorated quasicrystals and their diffraction patterns
}

\begin{tabular}{|c|c|}
\hline Journal: & Philosophical Magazine \& Philosophical Magazine Letters \\
\hline Manuscript ID: & TPHM-05-May-0223.R1 \\
\hline Journal Selection: & Philosophical Magazine \\
\hline $\begin{array}{r}\text { Date Submitted by the } \\
\text { Author: }\end{array}$ & 05-Jul-2005 \\
\hline Complete List of Authors: & $\begin{array}{l}\text { Kozakowski, Bartlomiej; AGH University of Science and Technology, } \\
\text { Faculty of Physics and Applied Computer Science } \\
\text { Wolny, Janusz; AGH University of Science and Technology, Faculty } \\
\text { of Physics and Applied Computer Science }\end{array}$ \\
\hline Keywords: & quasicrystals, diffraction \\
\hline Keywords (user supplied): & \\
\hline
\end{tabular}

\section{今 scholaroNE" \\ Manuscript Central}




\title{
Decorated quasicrystals and their diffraction patterns
}

\author{
B. Kozakowski, J. Wolny \\ Faculty of Physics and Applied Computer Science, AGH University of Science and \\ Technology, Kraków, Poland
}

PACS numbers: 61.44.Br, 61.43.-j, 61.10.Dp

Keywords: Quasicrystals, Diffraction pattern

\begin{abstract}
Structure factor of two-dimensional quasicrystals obtained for decorated Penrose tilings was derived in statistical approach. Each atom can decorate the two rhombuses in an arbitrary fashion with also arbitrary occupation probability. The derived analytical formula was numerically checked for refined model structure.
\end{abstract}

\section{Introduction}

For more than 20 years after the discovery of quasicrystals, the scientists are still facing problems concerning the determination of the structure of quasicrystals from the diffraction pattern. There are many different solutions and among them: the higher-dimensional analysis using "cut and project" method [1-5], clusters [6-8], approximants [9] and statistical approach [10-12]. In this paper we concentrate on the first and the last solutions. The higherdimensional analysis, although quite simple and clear, has some essential limitations, e.g.:

1) one has to know the atomic surface which can have very complicated and different shapes for every configuration of atoms,

2) similarly to above, one has to determine positions of atoms decorating $5 \mathrm{D}$ elementary unit cell, which after projection onto real space would give us the coordinates of atoms belonging to rhombuses or clusters. It is a very time-consuming process to decorate a $5 \mathrm{D}$ unit cell.

3 ) it is very hard to describe the dynamical properties of quasicrystals. Even thermal vibrations cause some trouble. For example, if atoms vibrate along $y$ direction, along which direction do they vibrate in 5D space?

4) an attempt to deal with defective structures brings us to very serious and unsolved problems.

The higher-dimensional analysis gave us though one fundamental result: a structure factor which perfectly describes ideal (and only ideal) quasicrystals. For the Penrose lattice the structure factor is:

$$
F\left(k_{\| x}, k_{\| y}\right)=\sum_{z=1}^{4} \exp \left(-\mathrm{i} k_{z} z\right) \int_{A S} \exp \left(-i\left(k_{\perp x} x_{\perp}+k_{\perp y} y_{\perp}\right)\right) \mathrm{d} x_{\perp} \mathrm{d} y_{\perp}
$$

where $A S$ stands for the atomic surface in perp-space.

Our goal is to derive a structure factor which should be equal to (1) for ideal quasicrystal and which would not have the limitations enumerated above. It would be possible for a method working in the physical space only and which would use a statistical distribution of atomic positions.

\section{Indexing}


For 5D representation of Penrose tiling one can write a scattering vector using 5 indices $\left(h_{1}, h_{2}, h_{3}, h_{4}, h_{5}\right)$ as following:

$$
\mathbf{K}=h_{1} \hat{\mathbf{x}}_{1}+h_{2} \hat{\mathbf{x}}_{2}+h_{3} \hat{\mathbf{x}}_{3}+h_{4} \hat{\mathbf{x}}_{4}+h_{5} \hat{\mathbf{x}}_{5} ; \quad \hat{\mathbf{x}}_{1}=[1,0,0,0,0] ; \ldots ; \hat{\mathbf{x}}_{5}=[0,0,0,0,1]
$$

In our work we choose another basis vectors, i.e. $\mathbf{K}_{\mathbf{1}}=[0,0,-1,-1,0], \mathbf{K}_{2}=[-1,-1,0,0,0]$ and $\tau-$ times $(\tau \approx 1.618)$ shorter modulation vectors $\mathbf{Q}_{1}=[1,0,0,0,0], \quad \mathbf{Q}_{2}=[0,0,0,1,0]$ and $\mathbf{Z}=[1,1,1,1,1]$. Then $\mathbf{K}=n_{1} \mathbf{K}_{\mathbf{1}}+n_{2} \mathbf{K}_{\mathbf{2}}+m_{1} \mathbf{Q}_{\mathbf{1}}+m_{2} \mathbf{Q}_{\mathbf{2}}+z \mathbf{Z}$. Having projected vector $\mathbf{K}$ on physical space we get:

$$
\mathbf{k}=n_{1} \mathbf{k}_{1}+n_{2} \mathbf{k}_{2}+m_{1} \mathbf{q}_{1}+m_{2} \mathbf{q}_{2}
$$

where $\mathbf{k}_{1}, \mathbf{k}_{2}, \mathbf{q}_{1}, \mathbf{q}_{2}$ are the real space components of $\mathbf{K}_{1}, \mathbf{K}_{2}, \mathbf{Q}_{1}, \mathbf{Q}_{2}$.

As we can see (3) doesn't depend on $\mathbf{Z}$ vector. That means, in order to index whole set of peaks we need only four vectors and four indices.

Vectors $\mathbf{k}_{\mathbf{1}}$ and $\mathbf{q}_{\mathbf{1}}$ are directed at the angle of $72^{\circ}$ and $\mathbf{k}_{\mathbf{2}}$ and $\mathbf{q}_{\mathbf{2}}$ at the angle of $-72^{\circ}$ to the $x$ axis and $\left|\mathbf{k}_{\mathbf{1}(2)}\right| \approx 4.067$ and $\left|\mathbf{q}_{1(2)}\right|=\left|\mathbf{k}_{\mathbf{1}(2)}\right| / \tau \approx 1.553$

An arbitrary diffraction peak for scattering vector $\mathbf{k}$ can be then expressed as a linear combination of $\mathbf{k}_{1(2)}$ and $\mathbf{q}_{1(2)}$ and its components $\left(k_{x}, k_{y}\right)$ are equal to:

$$
k_{x}=k_{0} c_{1}\left(n_{x}+m_{x} / \tau\right) ; \quad k_{y}=k_{0} s_{1}\left(n_{y}+m_{y} / \tau\right)
$$

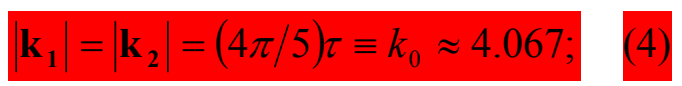

where: $c_{p}=\cos (2 \pi p / 5), s_{p}=\sin (2 \pi p / 5), n_{\mathrm{x}} \equiv n_{1}+n_{2}, \quad n_{\mathrm{y}} \equiv n_{1}-n_{2}, \quad m_{\mathrm{x}} \equiv m_{1}+m_{2}, \quad m_{\mathrm{y}} \equiv m_{1}-m_{2}$ More information concerning the indexing can be found in [11]

\section{Statistical approach}

In higher dimensional representation diffraction peaks are given by the relation: $\exp (\mathbf{i K} \cdot \mathbf{R})=1$, what leads to:

$$
\mathbf{K} \cdot \mathbf{R}=\mathbf{k}_{\|} \cdot \mathbf{r}_{\|}+\mathbf{k}_{\perp} \cdot \mathbf{r}_{\perp}=k_{x} u_{x}+k_{y} u_{y}+k_{\perp x} x_{\perp}+k_{\perp y} y_{\perp}+k_{z} z=2 \pi \alpha
$$

where $\left(u_{x}, u_{y}\right)$ are reduced $(x, y)$ components, such that $(\mathbf{k} \cdot \mathbf{r} \bmod 2 \pi)=\mathbf{k} \cdot \mathbf{u}$. As an integer multiple of $2 \pi$ doesn't affect the structure factor calculations, one can choose the $\alpha$ parameter equal to zero. For main reflections only, i.e for $m_{1(2)}=0$, we can rewrite (5) as:

$$
u_{x}=-\frac{k_{\perp x}}{k_{x}} x_{\perp}-\frac{k_{z}}{k_{x}} z ; \quad u_{y}=-\frac{k_{\perp y}}{k_{y}} y_{\perp}
$$

Then the structure factor (1) reads:

$$
F_{n}=\sum_{z=1}^{4} \int_{S} \exp \left(\mathrm{i}\left(n_{x} k_{0} c_{1} u_{x}+n_{y} k_{0} S_{1} u_{y}+z k_{z} n_{x}\right)\right) \mathrm{d} u_{x} \mathrm{~d} u_{y}
$$

It looks very similar to (1) but this time the Fourier integral is over $S$, i.e. the area in physical space obtained after projection of atomic surface $A S$ according to (6). Very similar calculations performed for a satellite reflection indexed by $m_{1(2)}$ gives:

$$
u_{x}^{\prime}=-\frac{q_{\perp x}}{q_{x}} x_{\perp}-\frac{q_{z}}{q_{x}} z ; \quad u_{y}^{\prime}=-\frac{q_{\perp y}}{q_{y}} y_{\perp}
$$


and

$$
F_{m}=\sum_{z=1}^{4} \int_{S^{\prime}} \exp \left(\mathrm{i}\left(m_{x} q_{0} c_{1} u_{x}{ }^{\prime}+m_{y} q_{0} s_{1} u_{y}{ }^{\prime}+z m_{x} q_{z}\right)\right) \mathrm{d} u_{x}{ }^{\prime} \mathrm{d} u_{y}{ }^{\prime}
$$

Equation (7) works only for $\mathbf{k}=n_{1} \mathbf{k}_{1}+n_{2} \mathbf{k}_{2}$ and (9) only for $\mathbf{k}=m_{1} \mathbf{q}_{1}+m_{2} \mathbf{q}_{2}$. It is so because we integrate over different areas $\left(S\right.$ and $\left.S^{\prime}\right)$. If we need to describe every diffraction peak (3), we should transform $S$ ' into $S$. Once we have one common area $S$ we can calculate Fourier transform for any $\mathbf{k}(3)$. Luckily there is a very simple relation between these areas. Almost all we need to do is to divide (8) by (6) and we get $u_{\mathrm{x}}{ }^{\prime}=-\tau^{2} u_{\mathrm{x}}, u_{\mathrm{y}}{ }^{\prime}=-\tau^{2} u_{\mathrm{y}}$, what leads us to a general expression for the structure factor

$$
F_{n, m}=\sum_{z=1}^{4} \int_{S} \exp \left(\mathrm{i}\left(w_{x} u_{x}+w_{y} u_{y}+\varphi_{z}\right)\right) \mathrm{d} u_{x} \mathrm{~d} u_{y}
$$

where $\varphi_{z}=-z\left(k_{z} n_{x}+q_{z} m_{x}\right)$ and $w_{x}=c_{1} k_{0}\left(n_{x}-\tau m_{x}\right) ; \quad w_{y}=s_{1} k_{0}\left(n_{y}-\tau m_{y}\right)$

Because now we are using only physical coordinates $\left(u_{x}, u_{y}\right)$, the $S$ area can be considered as probability distribution of atoms' positions belonging to the Penrose lattice. Doing some statistical research we already showed that [13] that $S$ consists of four pentagons. Now we are showing that these pentagons are just a projection (6) of the $A S$ onto the real space. It means that the eg. (10) is, for the ideal Penrose lattice, equivalent to (1). Equation (10) has but one strong advantage over (1): quasicrystal doesn't have to be ideal any more. We can add thermal vibration, same defects and (10) will be fine, because all those things we can take into account by changing or smoothing (additional probability function is then needed $\mathrm{P}\left(u_{x}, u_{y}\right)$ - for further details see [11]) the $S$ area. Notice also we haven't made any assumption of projecting area. It can be the whole $A S$ but it can also be only a part of it - for instance, an area corresponding to a local configuration of atoms, which after projection would build thick or thin rhombus (figures 1 and 2 explain the way of doing this)

\section{Decorated structures}

Eg. (10) is derived for a particular set of atoms. What would happen, if we had $N$ such identical sets and they would be shifted by $\mathbf{r}_{\mathrm{j}}$ vector? We would take this shift into account by multiplying (10) by $\exp \left(\mathbf{k}_{\|} \cdot \mathbf{r}_{\mathrm{j}}\right)$. Of course, we don't want to move the whole Penrose lattice. We need to divide the Penrose lattice into sets of thick and thin rhombuses and then to find the parts of atomic surface belonging to both of these sets. Finally we project these sets onto real space, and for one of these sets (for instance for thick rhombuses) we get:

$$
F\left(\mathbf{k}_{\|}\right)=\sum_{z=1}^{4} \sum_{j=1}^{N} \exp \left(\mathrm{i}\left(\mathbf{k}_{\|} \cdot \mathbf{r}_{j}\right)\right) \int_{S_{L}} \exp \left(\mathrm{i}\left(w_{x} u_{x}+w_{y} u_{y}+\varphi_{z}\right)\right) \mathrm{d} u_{x} \mathrm{~d} u_{y}
$$

The most important point of presented analysis is the determination of the probability distributions for particular point belonging to the $\mathrm{L}$ and $\mathrm{S}$ rhombuses (i.e. $S_{\mathrm{L}}$ and $S_{\mathrm{S}}$ ). Two rhombuses were presented in figure 1 . For our convenience we placed both the A points of thick and thin rhombuses in the origin of the coordinate system. In figure 2 the appropriate distributions were shown for the A point belonging to the thick rhombus in orientation as shown in figure 1 . The positions of vertices $\mathrm{A}, \mathrm{B}, \mathrm{C}$ and $\mathrm{D}$ on the atomic surface were shown in figure 2(a). The real space vector $\mathbf{A B}$ was also shown in perp-space in figure 2(c). One has to find a distribution of positions of $\mathrm{A}$ atoms on small pentagon at $z=1$, for which atom $\mathrm{B}$ lies on the pentagon at $z=2$. Simply construction shows that required distribution is bounded by trapezoid (greyed area in figure 2(b)). The next construction for vectors AC was shown in figures 2(c) and that leads to triangular distribution marked in the figure (dark grey). One can also notice that the last vector AD doesn't change the obtained previously triangular 
distribution. Similar constructions for thin rhombus also gives a triangular distribution. Having calculated structure factor for thick $\left(F_{L}\right)$ and thin $\left(F_{S}\right)$ rhombuses as a Fourier transform of the appropriate triangular distributions one can write the structure factor for decorated Penrose tiling as

$$
\frac{F=C \cdot \operatorname{re}\left(\sum_{\alpha=1}^{5}\left(F_{L} \sum_{l=1}^{n_{L}} p_{l} \exp \left(\mathbf{i} \mathbf{k}_{\|} \cdot \mathbf{r}_{l}\right)+F_{S} \sum_{s=1}^{n_{S}} p_{s} \exp \left(\mathbf{i} \mathbf{k}_{\|} \cdot \mathbf{r}_{\mathbf{s}}\right)\right)\right)}{F_{L(S)}\left(\mathbf{k}_{\|}\right)=\sum_{j=1}^{N_{L(S)}} \int_{S_{L(S)}} \exp \left(\mathrm{i}\left(w_{x} u_{x}+w_{y} u_{y}+\varphi_{1}\right)\right) \mathrm{d} u_{x} \mathrm{~d} u_{y}}
$$

where sum over $\alpha$ is for five different directions, $p_{l(s)}$ are the occupation probabilities and $C$ is a normalizing constant value such that $F(0,0)=1$. In principle there are ten orientations, but due to the inversion one can use only five of them and the real part of the Fourier transform. Parameters $p_{L(\mathrm{~S})}$ are very important for disordered structures and also for decorating atoms placed at the edges of rhombuses (see the example below). We also neglected the sum over $z$ because both $S_{\mathrm{L}}$ and $S_{\mathrm{S}}$ are projected from the same pentagon which lays in the hyperspace at $z=1$. More details of the calculations including rotations of triangular distributions for particular decorating atoms are given in [11].

\section{Example}

As an example, the structure factor (12) was used for refining a model structure, which was the perfect Penrose tiling. For refining process we took about 2000 numerically calculated (from a set of about 150000 points belonging to Penrose lattice) diffraction peaks and $I_{\min } / I_{\max }=0.001$. We wanted to get probability values of rhombuses' vertices occupation. Using the least square method six parameters were refined. The refining was started from the uniform distribution and the procedure (based on gradient method) converged very rapidly to the theoretical values. After several steps (about ten) the results of fitting were quite acceptable, with the $R$-factor reaching a few percents. And it was only about 50 iterations when the theoretical values were recovered This example shows two important things: first: it supports the correctness of the obtained formula for the structure factor (12) and second: it indicates that very conventional refining methods, well elaborated in classical crystallography, can be directly adapted to refining process of quasicrystals.

\section{Conclusions}

In this paper we have thoroughly discussed the structural properties of twodimensional quasicrystals. Using the projection method we were able to derive a probability distribution of chosen atom (in our case placed at the origin in figure 1) belonging to thick or thin rhombuses of the Penrose tiling. A physical space calculations allowed us to write an analytical formula for any decorated structure with arbitrary probabilities of atomic sites' occupation. In the discussed example it was shown that all the well-known methods of structure refinement can be also adapted for quasicrystals. The discussed method of structure factor calculation was also successfully applied to the cluster approach [12].

The authors would like to acknowledge the State Committee of Scientific Research for financial support. 


\section{References}

[1] N.G. de Bruijn, Proc. K. Ned. Akad. Wet. Ser. A 4339 (1981).

[2] M. Duneau, A. Katz, Phys. Rev. Lett. 542688 (1985).

[3] P. Kramer and R. Neri, Acta Crystallogr. A 40580 (1984).

[4] V. Elser, Phys. Rev. B 314892 (1985).

[5] A. Yamamoto, Phys. Rev. Lett. 93 art. no. 195505 (2004).

[6] P.J. Steinhardt P.J. and H.-C. Jeong, Nature 382433 (1996).

[7] P. Gummelt, Geometriae Dedicata 621 (1996).

[8] Coverings of Discrete Quasiperiodic Sets, Theory and Applications to Quasicrystals, edited by P. Kramer and Z. Papadopolos, Springer Tracts in Modern Physics, SpringerVerlag Berlin Heidelberg, 2003.

[9] W. Steurer, Ferroelectrics 250377 (2001)

[10] J. Wolny, Philosophical Magazine A 77395 (1998).

[11] B. Kozakowski and J. Wolny, Structure factor for decorated Penrose tiling. http://arxiv.org/ftp/cond-mat/papers/0503/0503464.pdf, (2005).

[12] A. Dąbrowska, B. Kozakowski and J. Wolny, Acta Crystall. A 61350 (2005).

[13] J. Wolny, B. Kozakowski, P. Repetowicz, J. Alloys and Compounds 342 (2002) 198-202. 


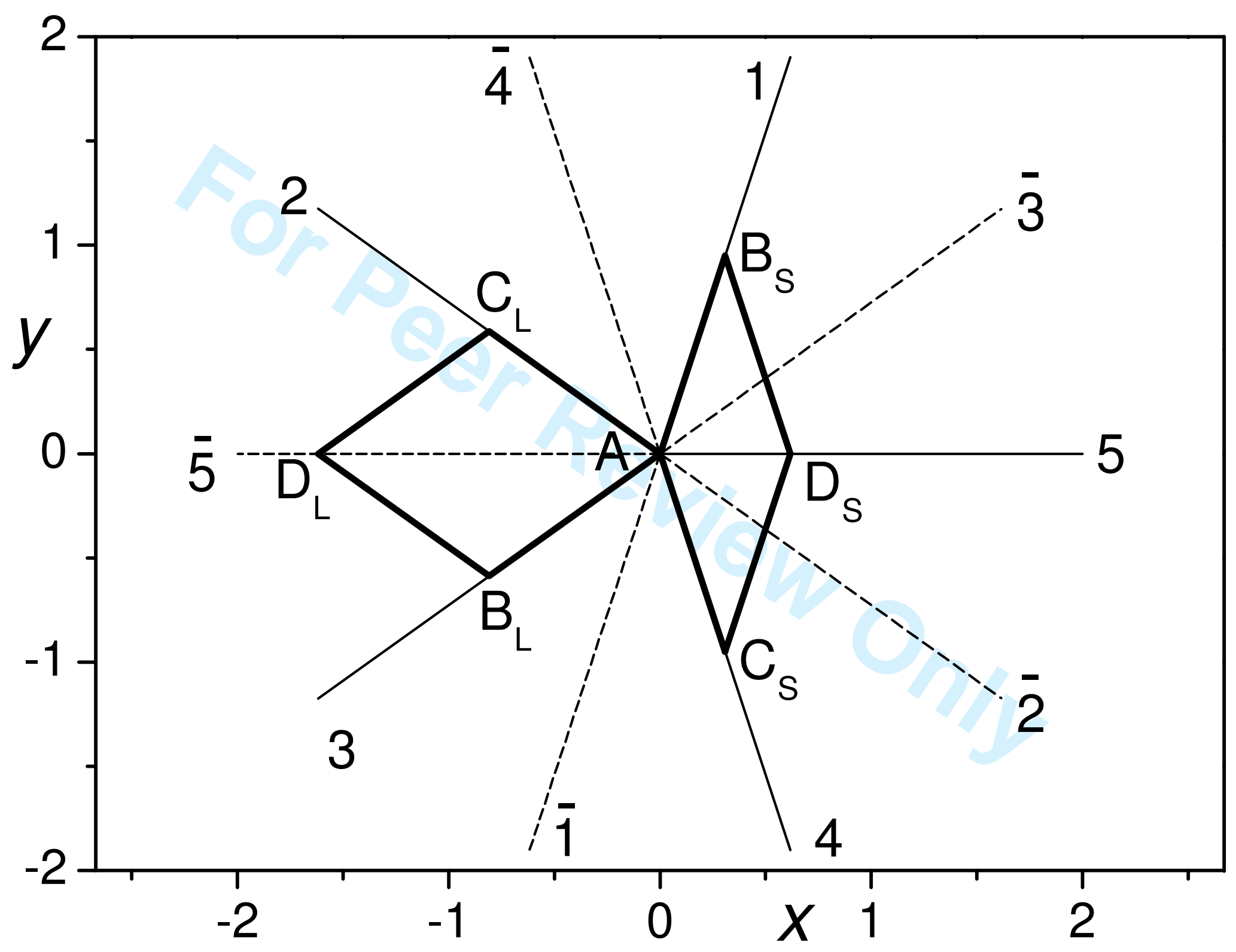

http://mc.manuscriptcentral.com/pm-pml 
Page 7 of 7

Philosophical Magazine \& Philosophical Magazine Letters

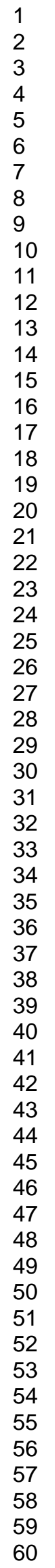
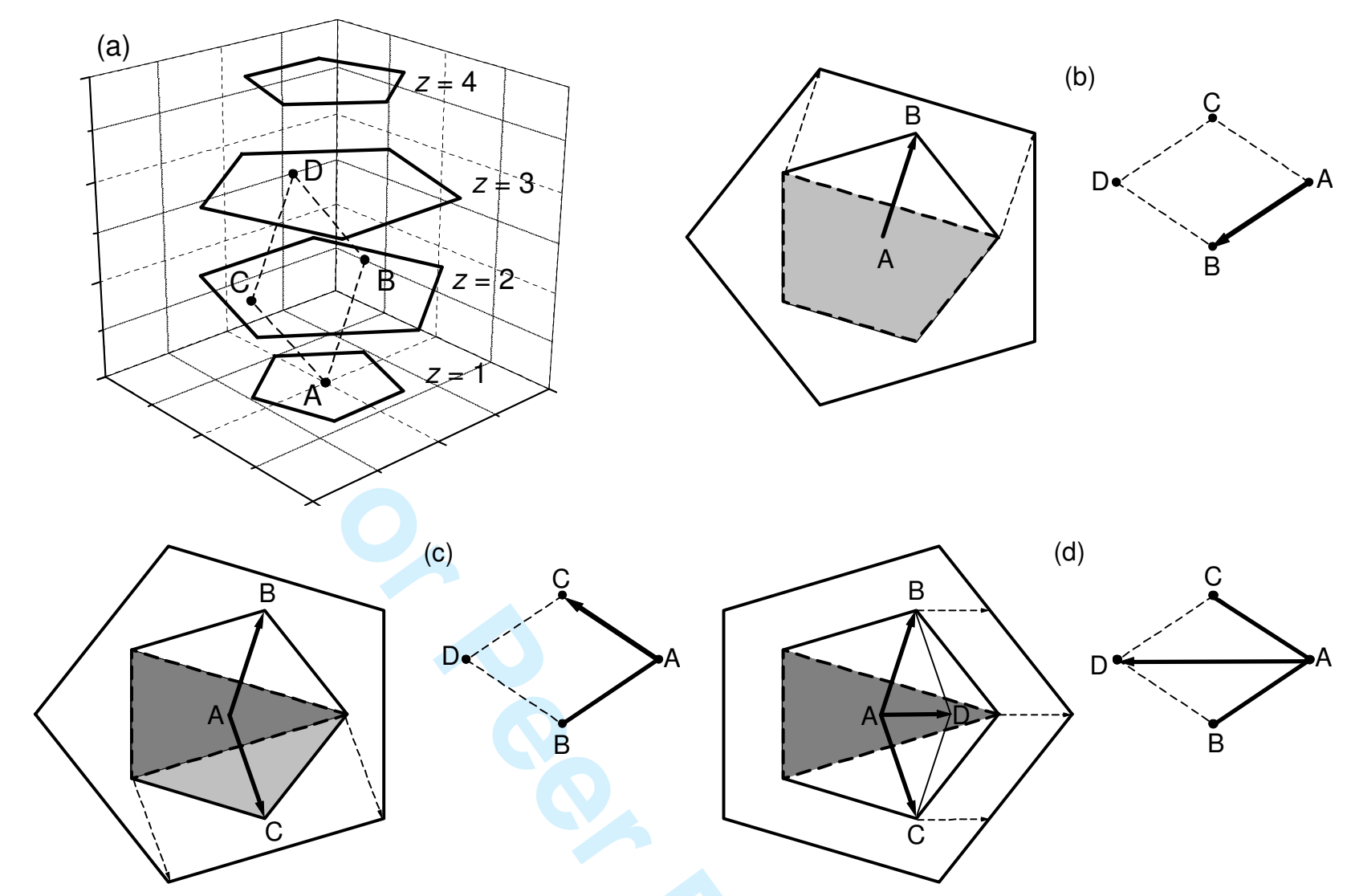

(d)

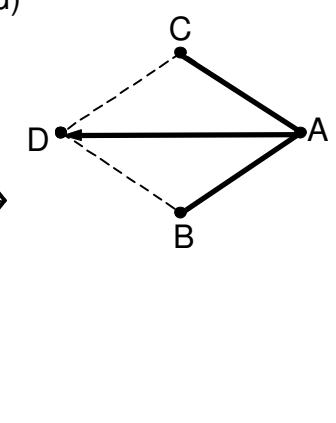

http://mc.manuscriptcentral.com/pm-pml 